\title{
Relativistic electron acceleration and decay time scales in the inner and outer radiation belts: SAMPEX
}

\author{
D. N. Baker, ${ }^{1}$ J. B. Blake, ${ }^{2}$ L. B. Callis,${ }^{3}$ J. R. Cummings, ${ }^{4}$ D. Hovestadt, ${ }^{5}$ \\ S. Kanekal,${ }^{1}$ B. Klecker, ${ }^{5}$ R. A. Mewaldt, ${ }^{4}$ and R. D. Zwick ${ }^{6}$
}

Abstract. High-energy electrons have been measured systematically in a low-altitude $(520 \times 675 \mathrm{~km})$, nearly polar (inclination $=82^{\circ}$ ) orbit by sensitive instruments onboard the Solar, Anomalous, and Magnetospheric Particle Explorer (SAMPEX). Count rate channels with electron energy thresholds ranging from $0.4 \mathrm{MeV}$ to $3.5 \mathrm{MeV}$ in three different instruments have been used to examine relativistic electron variations as a function of $L$-shell parameter and time. A long run of essentially continuous data (July 1992 July 1993) shows substantial acceleration of energetic electrons throughout much of the magnetosphere on rapid time scales. This acceleration appears to be due to solar wind velocity enhancements and is surprisingly large in that the radiation belt "slot" region often is filled temporarily and electron fluxes are strongly enhanced even at very low Lvalues $(L \sim 2)$. A superposed epoch analysis shows that electron fluxes rise rapidly for $2.5 \leq \mathrm{L} \leq 5$. These increases occur on a time scale of order 1-2 days and are most abrupt for $L$-values near 3 . The temporal decay rate of the fluxes is dependent on energy and L-value and may be described by $J$ $=\mathrm{Ke}-\mathrm{t} /$ to with $\mathrm{t}_{\mathrm{o}}=5-10$ days. Thus, these results suggest that the Earth's magnetosphere is a cosmic electron accelerator of substantial strength and efficiency.

\section{Introduction}

It is of great interest in magnetospheric physics to understand basic particle acceleration and loss mechanisms and to determine the variation time scales for high-energy electrons throughout the terrestrial radiation belts. It has been suggested that relativistic electrons could provide a significant coupling mechanism between the magnetosphere and the Earth's middle atmosphere (Baker et al., 1987; Callis et al., 1991). Relativistic electrons in the Earth's magnetosphere are also of considerable practical importance because of their deleterious effect on spacecraft subsystems (Gussenhoven et al., 1987).

Relatively few studies have been able to examine the long-term variation of magnetospheric electrons over a broad range of energies and magnetic $L$-shell parameters.

\footnotetext{
${ }_{1}^{1}$ ASA/Goddard Space Flight Center, Greenbelt, MD

2 Aerospace Corporation, El Segundo, CA

3NASA/Langley Research Center, Hampton, VA

${ }^{4}$ California Institute of Technology, Pasadena, CA

${ }^{5}$ Max Planck Institut fur Extraterrestrische Physik

6NOAA/Space Environment Laboratory, Boulder, CO

Copyright 1994 by the American Geophysical Union.
}

Paper number 93GL03532

$0094-8534 / 94 / 93 G L-03532 \$ 03.00$
Gussenhoven et al. (1987) showed the average pattem of $E \geq$ 1.0 MeV electrons using dosimeters onboard the Defense Meteorological Satellite Program (DMSP) spacecraft at 840 $\mathrm{km}$ altitude; these authors saw evidence of 27-day recurrences of flux enhancements throughout one year (1984) of their observations (see, also, Williams, 1966). Other authors such as Kohno et al. (1990) have mapped the average intensities of high-energy electrons (0.19-3.2 MeV) using the OHZORA (EXOS-C) spacecraft; their results were averaged over several years and consequently did not reveal details of periodicity or enhancement and decay timescales. Recent studies (e.g., Imhof et al., 1991) have been able to examine several months of low-altitude electron $(E>1.0$ $\mathrm{MeV}$ ) data and compare this with $\mathrm{L}=6.6$ measurements. Imhof et al. found a good correlation between intense precipitation spikes at low altitude and trapped flux levels at geostationary orbit.

We present new measurements of relativistic electron flux variations as determined with sensitive sensor systems onboard the Solar, Anomalous, and Magnetospheric Particle Explorer (SAMPEX). We report on approximately the first year of data using several different energy thresholds and covering all relevant L-values. The data show abrupt electron enhancements throughout the magnetosphere on time scales of a day or two. This suggests a remarkably coherent global behavior for electron acceleration. We conclude that the observed large flux enhancements are due to strong solar wind streams impinging on the magnetosphere and these results are indicative of a cosmic acceleration mechanism of considerable strength and efficiency.

\section{Instrumentation and Data Analysis}

The SAMPEX spacecraft was launched from the Western Test Range at 1419 UT on 3 July 1992. It achieved the designed orbit altitude of $520 \times 675 \mathrm{~km}$ with an orbital inclination of $81.7^{\circ}$. The SAMPEX payload contains four separate instruments which generally point toward the zenith over the poles of the Earth. The overall configuration of the SAMPEX spacecraft, the orbital pointing strategy, and details of the instruments may be found in Baker et al. (1993) and associated papers.

The Proton/Electron Telescope (PET) measures 0.4 - 30 $\mathrm{MeV}$ electrons (see Cook et al., 1993 for details). One of the PET count rates corresponds to a singles rate (P1) from a 2 mm thick detector which has a threshold of $400 \mathrm{keV}$. (This rate also responds to $>4 \mathrm{MeV}$ protons, but other measurements show that electrons almost always dominate the P1 rate in the outer zone.) Two other coincidence rates in PET (ELO, EHI) correspond to channels that are clearly identified as electrons. ELO responds effectively to $2.1-6.0$ 
$\mathrm{MeV}$ electrons, while EHI responds to $3.5-15 \mathrm{MeV}$ electrons. All of the PET rate data used here were acquired at 6-s resolution.

The other SAMPEX sensor used in this study is the Heavy Ion Large Telescope (HILT). HLT is a large ion drift chamber-proportional counter with solid-state detector (SSD) elements at the back of the telescope (see Klecker et al., 1993). Although these detectors are also sensitive to $\geq 4$ $\mathrm{MeV}$ protons, at mid-latitudes the HILT SSDs respond almost exclusively to energetic electrons. Two thresholds ( $E>1 \mathrm{MeV}$ and $\mathrm{E}>3 \mathrm{MeV}$ ) are set electronically for the HILT SSDs and the 16 individual elements give a very large $(60 \mathrm{~cm} 2-\mathrm{sr})$ geometric factor.

\section{Observations}

Prior long-term studies of relativistic electron variations in the magnetosphere have often focused upon the continuous measurements available from geostationary orbit (e.g., Baker et al., 1986; 1987). Figure 1 shows in the bottom panel the daily average flux of $>2 \mathrm{MeV}$ electrons measured at $6.6 \mathrm{R}_{\mathrm{E}}$ by sensors on the GOES-7 spacecraft. The data cover the period July 1992 through February 1993, and show the kind of large, recurrent peaks in flux that are characteristic of the period approaching the solar activity minimum (Baker et al., 1986). The large flux enhancements

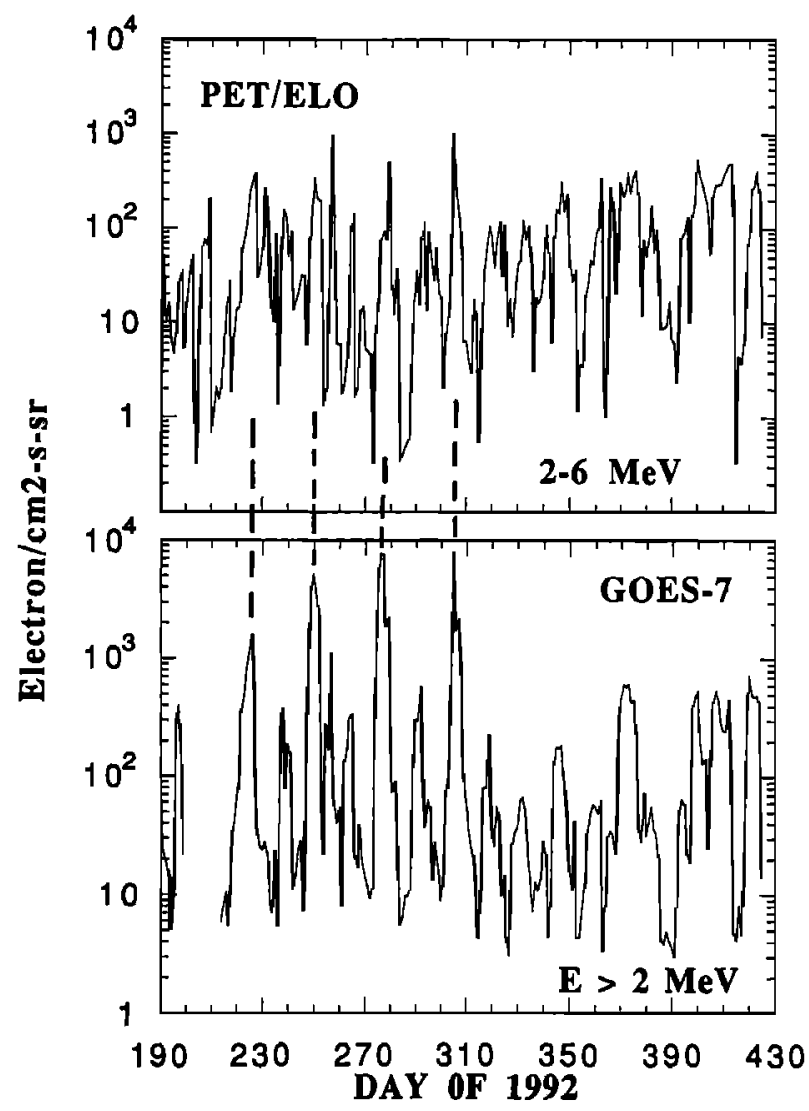

Fig. 1. (Bottom) The $\mathrm{E}>2 \mathrm{MeV}$ electron fluxes measured from July 1992 through February 1993 by the GOES-7 spacecraft at geostationary orbit. (Top) Electron fluxes measured by the PET sensor on SAMPEX in the energy range $2-6 \mathrm{MeV}$ in the low altitude range $6 \leq \mathrm{L} \leq 7$. at $6.6 \mathrm{R}_{\mathrm{E}}$ typically last several days and correspond to increases in absolute intensity of more than two orders of magnitude. Examination of concurrent IMP-8 solar wind data (courtesy of A. Lazarus) shows that the GOES-7 electron enhancements are associated with high speed solar wind streams in which the velocity rapidly rises to $\geq 600$ $\mathrm{km} / \mathrm{s}$. Hence, the relativistic electron events in 1992-93 are closely analogous to similar events observed during the last solar cycle during 1983-85 (Baker et al., 1986). The upper panel of Figure 1 shows daily averages of the 2-6 MeV electron flux from the SAMPEX PET/ELO sensor for the Lvalue range $6.0-7.0$. It is evident that there is a temporal correspondence between the enhancements at GOES-7 and those observed at the same general L-value by SAMPEX.

A much more comprehensive view of the SAMPEX electron measurements is provided in Figure 2. Here the P1 counting rate data, sorted according to L-value (in bins of $0.1 \mathrm{~L}$ ), are plotted versus day of year. The logarithm of the P1 ( $\mathrm{E}>0.4 \mathrm{MeV}$ ) electron intensity level in each L-bin has been color-coded according to the color bar to the right of the figure. L-values ranging from 1.0 to 11.0 are shown. Note the numerous sharp flux increases that occur in the outer zone $(L \approx 4.0)$ throughout the period shown. Examples of this are seen at Day 220, Day 248, Day 275, etc. The abrupt enhancements occur in about one day, extending over a broad range in $L$, evidently reaching to high latitudes and often going beyond $L \approx 6$. These flux increases evidently correspond to the relativistic electron enhancements seen at GOES-7 (see Figure 1).

What is perhaps more notable, however, is that the same flux enhancements extend to relatively low L-values. Note in Figure 2 that most sharp enhancements at $\mathrm{L} \sim 4.0$ also correspond to abrupt increases extending down to $L \leq 3.0$. Even the magnetospheric "slot" region around L $~ 2.5$ which separates the inner and outer zones is often temporarily filled with electrons. Previous results (Vampola, 1971) suggested that the slot region is normally filled only during major geomagnetic storms. The SAMPEX data show more frequent effects: One major solar particle event occurred in the period under discussion (see the filled polar cap region after Day 304), but essentially all other events in the data record are due to high-speed solar wind streams.

It could be argued that the effects seen in Figure 2 for SAMPEX might be due to changes in equatorial pitch angle distributions that lower the electron mirror points down to SAMPEX altitudes $(\sim 600 \mathrm{~km})$. However, earlier data from the Combined Release and Radiation Effects Satellite (CRRES) very near the equatorial plane (see Blake et al., 1992 and references therein) showed closely analogous effects. The CRRES spacecraft was in an $18^{\circ}$-inclination geostationary transfer orbit $\left(300 \mathrm{~km} \times 6.6 \mathrm{R}_{\mathrm{E}}\right)$ and it operated from July of 1990 to October of 1991 . Figure 3 shows data from the RE2 channel of the scatter detector onboard CRRES which measured electrons with $E>460$ $\mathrm{keV}$. The log of the flux is color-coded as shown at the right of the figure and the data are binned in L-value for each 12 hr orbit. As in Figure 2, numerous examples of abrupt electron enhancements extend across all $\mathrm{L}$-values from $\mathrm{L} \sim 2$ to $L \geq 6$. The enhancements often fill the equatorial slot region as seen in the SAMPEX data of Figure 2.

We have also examined both the $>1 \mathrm{MeV}$ and the $>3$ $\mathrm{MeV}$ channels from HILT using the same L-binning 


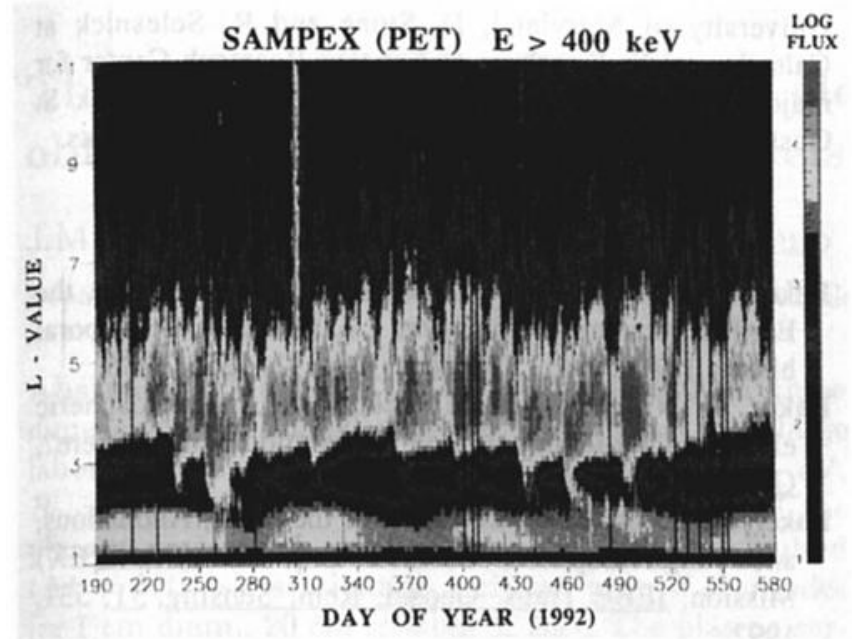

Fig. 2. A color-coded representation of electron fluxes measured by the SAMPEX P1 channel ( $~ 20.4 \mathrm{MeV}$ ) in PET from July of 1992 to July 1993. The data are binned in 0.1 L-values and the range $1 \leq \mathrm{L} \leq 11$ is shown.

procedure. Most of the same features show up in those data. Figure 4, for example, shows the color-coded data for the HILT > $1 \mathrm{MeV}$ electrons. The plot shows quite prominently the abrupt enhancements of flux in the outer zone and also shows several examples of filling in of the slot region as was seen in Figures 2 and 3 above. (Note that a protective door on the HILT sensor was closed during the period from launch to Day 232 and thus the electrons penetrating the door were of much higher incident energy early in the mission.).

Given the abrupt flux enhancements seen at $L \leq 4.0$ (see Figures 2-4), it is reasonable to define a set of days on which relativistic electron fluxes suddenly increase. This set of enhancement days can be taken as fiducial times for a superposed epoch analysis. We have taken the eight clearest increases seen near $L=3.0$ in the P1 channel. A zero epoch for these events was established as the peak counting rate day and the log of the fluxes ranging from Day $=-10$ to Day $=+19$ were then plotted. After performing this analysis

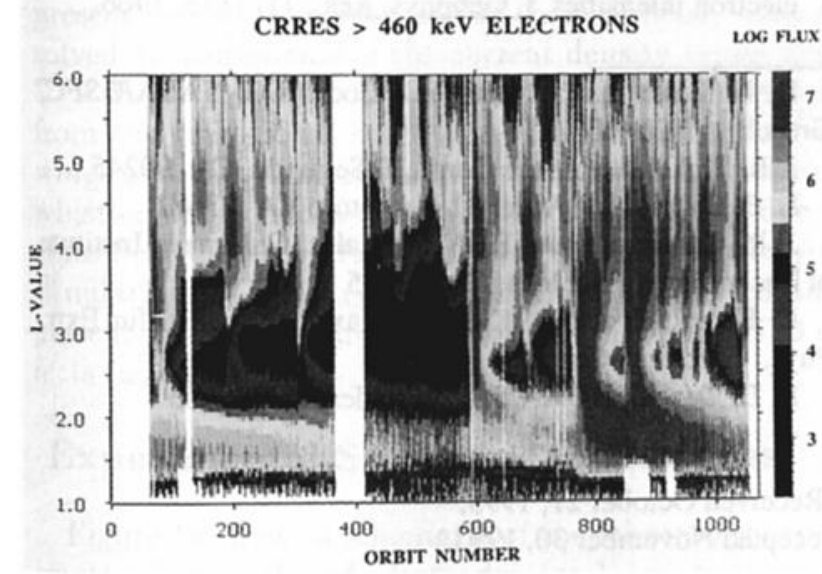

Fig. 3. Data similar to Fig. 2 , but for $>460 \mathrm{keV}$ electrons measured near the magnetic equator by the CRRES spacecraft. Counting rates are plotted versus orbit number and the data cover the period July 1990 to October 1991.

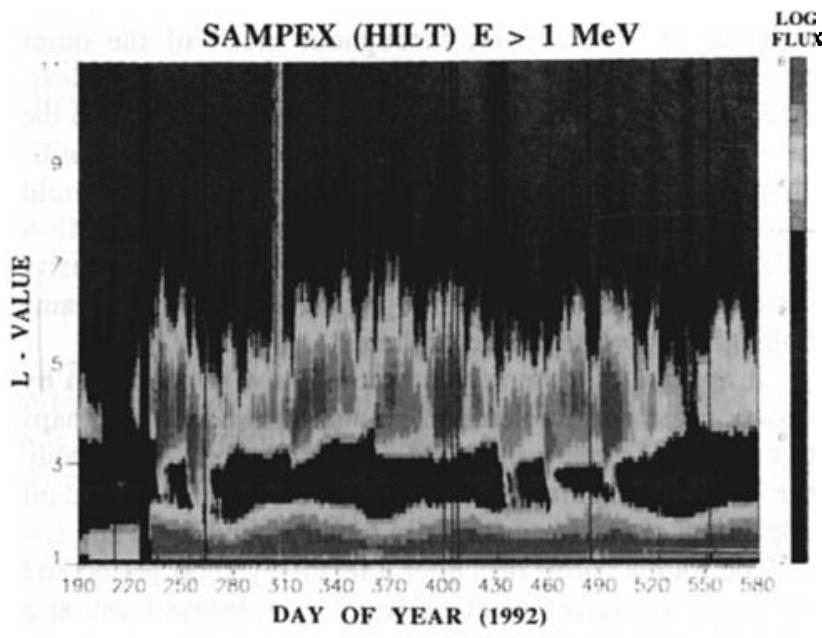

Fig. 4. Similar to Fig. 2 but showing SAMPEX electron fluxes for $\mathrm{E}>1 \mathrm{MeV}$ from the HILT instrument.

for $L=3.0$, the same fiducial days were used for $L=2.5,4.0$ and 5.0, and the superposed data from Day $=-10$ to +19 for each $\mathrm{L}$ values were plotted in the form of $\log$ of flux versus epoch day. In order to further compare the results, the data were normalized so that the highest peak value in each epoch set was assigned value 1.0. All epoch curves were then averaged together to form the superposed epoch plots shown in Figure 5. The data exhibit rather similar rise characteristics but decay characteristics are different depending on $\mathrm{L}$-value. A reasonable flux decay description is given by $\mathrm{J}=\mathrm{K} \exp \left(-\mathrm{t} / \mathrm{t}_{\mathrm{o}}\right)$ with $5 \leq \mathrm{t}_{0} \leq 10$ day. The value found for $t_{0}$ in the slot region $(\mathcal{L} \approx 2.5)$ is considerably smaller than for $L \geq 3$.

\section{Discussion and Conclusions}

Observations presented in this paper reveal that relativistic electrons increase in absolute intensity, often by

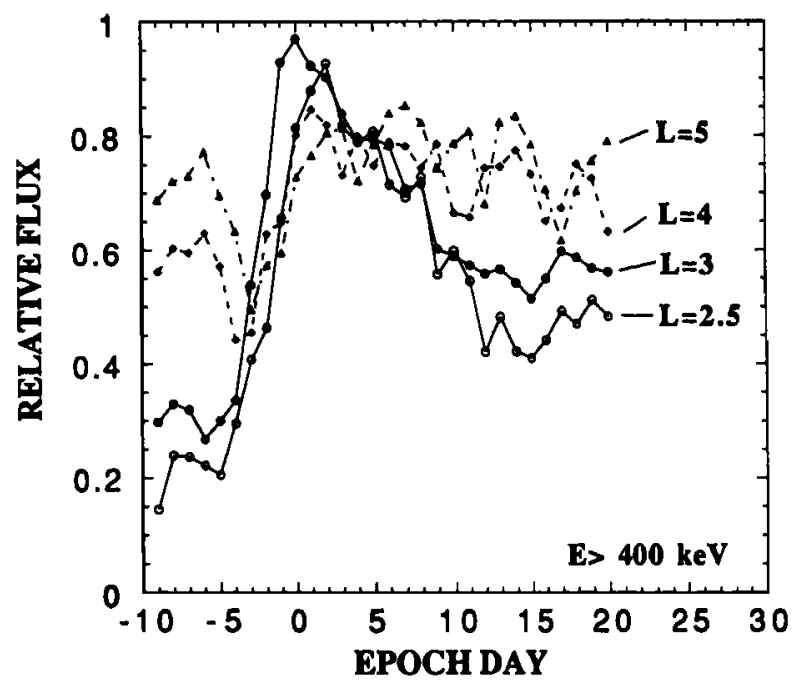

Fig. 5. A superposed epoch analysis of electron increases and decay rates for several selected L-values and for electron energies $>400 \mathrm{keV}$. The zero epoch days were 237,258 , 316 , and 366 of 1992 and days 33,71, 98, and 131 of 1993. 
a factor of 10 or more, throughout much of the outer magnetosphere on a time scale of order 1-2 days or less. Abrupt flux enhancements might be expected to occur in the outer magnetosphere, but it is more remarkable that low Lshells, deep within the magnetospheric cavity, would respond so prominently. As noted previously, the MMP-8 solar wind data show that the larger electron intensity increases are associated with high-speed solar wind streams impinging upon the magnetosphere.

It appears that the region below $L \approx 4$ is populated by occasional strong acceleration events, corresponding perhaps to a period of greatly enhanced radial diffusion. Presumably the source population is comprised of the abundant population of electrons normally found around $L=4$ and $L=$ 5. The source "tums off" after a relatively short period and the newly energized electron population decays away at a given location. Because another enhancement may not occur for weeks, a slot region is created as the electron intensities drop very low. Although the electron intensities between $L$ $\approx 4$ and $L=5.5$ wax and wane and the location of the peak intensity varies in radial distance, the source mechanisms are able to maintain a relatively intense energetic electron population.

Once the electrons are accelerated at a given magnetospheric position, their subsequent behavior at that location is dominated by radial diffusion, pitch angle diffusion, and ultimately atmospheric loss. From the color diagrams in Figures 2-4, we see some obvious evidence of radial diffusion: Such diffusion is manifested by a highintensity "pulse" of electrons (i.e., yellow and/or red "patches" in the color plots) moving gradually toward lower L-values. We see examples of this kind of behavior near $\mathrm{L}$ - 3 for Days 250-260 and again around Day 435 and Day 460 in Figures 2 and 4. However, in other cases the time variability of the electron fluxes seems to be dominated by an exponential decay at a given L-value. We interpret this latter feature as corresponding dominantly to an atmospheric loss process after the initial acceleration.

Given the evidence for relatively rapid decay of the relativistic electron fluxes, and given the probability that a major effect is loss into the atmosphere, this means that we should be able to analyze quantitatively the global atmospheric source strength for relativistic electron precipitation (see Baker et al., 1987; Callis et al., 1991). In light of our finding that characteristic electron lifetimes are of order a week in most regions of the magnetosphere, the electron population that is accelerated by passing solar wind streams must represent a strong input source throughout the entire band of atmospheric latitudes that map to the outer radiation zone. Even the inner zone electrons may sometimes contribute significantly. We look forward to a quantitative analysis of this interesting solar-terrestrial linkage issue.

Acknowledgments. We thank the many individuals who have contributed so extensively to the success of SAMPEX. We especially thank G. Mason and D. Hamilton at the
University of Maryland, E. Stone and R. Selesnick at Caltech, and R. Boughner at Langley Research Center for major contributions to this research. We also thank $S$. Gussenhoven of Phillips Laboratory for CRRES data plots.

\section{References}

Baker, D. N., et al., Highly relativistic electrons in the Earth's outer magnetosphere, l. Lifetimes and temporal history 1979-1984, J. Geophys. Res., 91, 4265, 1986.

Baker, D. N., et al., Highly relativistic magnetospheric electrons: A role in coupling to the middle atmosphere?, Geophys. Res. Lett, 14, 1027, 1987.

Baker, D. N., et al., An overview of the Solar, Anomalous, and Magnetospheric Particle Explorer (SAMPEX) Mission, IEEE Trans. Geosci. Rem. Sensing, 31, 531, 1992.

Blake, J. B., et al., Injection of electrons and protons with energies of tens of MeV into L < 3 on 24 March 1991, Geophys. Res. Lett., 19, 821, 1992.

Callis, L. B., et al., Precipitating relativistic electrons: Their long-term effect on stratospheric odd nitrogen levels, I. Geophys. Res., 96, 2939, 1991.

Cook, W. R., et al., PET: A proton/electron telescope for studies of magnetospheric, solar, and galactic particles, IEEE Trans. Geosci. Rem. Sensing 31, 565, 1993.

Gussenhoven, M. S., et al., New low-altitude dose measurements, IEEE Trans. Nuc, Sci., NS-34, 676, 1987.

Imhof, W. L., Relativistic electron enhancements: Simultaneous measurements from synchronous and low altitude satellites, Geophys. Res. Lett., 18, 397, 1991.

Klecker, B., et al., HILT: A heavy ion large area proportional counter telescope for solar and anomalous cosmic rays, IEEE Trans. Geosci. Rem. Sensing, 31, 542, 1993.

Kohno, T., et al., Intensity maps of $\mathrm{MeV}$ electrons and protons below the radiation belt, Planet. Space Sci., 38 , 483, 1990.

Vampola, A. L., Electron pitch angle scattering in the outer zone during magnetically disturbed times, I. Geophys. Res., 76, 4685, 1971.

Williams, D. J., A 27-day periodicity in outer zone trapped electron intensities, J. Geophys, Res., 71, 1815, 1966.

D. N. Baker and S. Kanekal, Code 690, NASA/GSFC, Greenbelt, MD 20771

J. B. Blake, Aerospace Corp., El Segundo, CA 90245

L. B. Callis, NASA/LaRC, Hampton, VA 23681

J. R. Cummings and R. A. Mewaldt, California Institute of Technology, Pasadena, CA 91125

D. Hovestadt and B. Klecker, Max Planck Inst. fur Extr. Phys., Garching, Germany

R. D. Zwickl, NOAA/SEL, Boulder, CO 80303

(Received October 21, 1993;

accepted November 30,1993.) 